\title{
ON CONLEY'S FUNDAMENTAL THEOREM OF DYNAMICAL SYSTEMS
}

\author{
M. R. RAZVAN
}

Received 25 February 2002

To the memory of Charles C. Conley

We generalize Conley's fundamental theorem of dynamical systems in Conley index theory. We also conclude the existence of a regular index filtration for every Morse decomposition.

2000 Mathematics Subject Classification: 37B25, 37B30, 37B35.

1. Introduction. Conley is mostly known for his fundamental theorem of dynamical systems and his homotopy index theory [1]. In the former, he proved that every continuous flow on a compact metric space admits a Lyapunov function which strictly decreases along nonchain recurrent orbits. This result has been developed by Franks for homeomorphisms [3] and Hurley for noncompact metric spaces [5, 6, 7, 8]. In the latter, Conley defined a homotopy invariant for any isolated invariant set for a continuous flow. This invariant gives some valuable information about the behavior of the isolated invariant set. This paper concerns a combination of these two masterpieces. Indeed, we show the existence of Conley's Lyapunov function on every index pair in the sense of Conley index theory. We also conclude the existence of a regular index filtration for every Morse decomposition.

2. Conley index theory. Let $\varphi^{t}$ be a continuous flow on a metric space $X$. An isolated invariant set is a subset $S \subset X$ which is the maximal invariant set in a compact neighborhood of itself. Such a neighborhood is called an isolating neighborhood. A Morse decomposition for $S$ is a collection $\left\{M_{i}\right\}_{i=1}^{n}$, where each $M_{i}$ is an isolated invariant subset of $S$ and for all $x \in S-\bigcup_{i=1}^{n} M_{i}$, there exist $i, j \in\{1, \ldots, n\}$ such that $i>j$, $\alpha(x) \in M_{i}$, and $\omega(x) \in M_{j}$. A pair $\left(A, A^{*}\right)$ of subsets of $S$ is called an attractor-repeller pair if $\left\{A, A^{*}\right\}$ is a Morse decomposition for $S$, that is, $\alpha(x) \in A^{*}$ and $\omega(x) \in A$ for every $x \in S-\left(A \cup A^{*}\right)$.

Let $S$ be an isolated invariant set with an isolating neighborhood $V$ and a Morse decomposition $\left\{M_{i}\right\}_{i=1}^{n}$. In [11], it is proved that if $\varphi^{[0,+\infty)}(x) \subset V$, then $\omega(x) \subset M_{i}$ for some $1 \leq i \leq n$. Similarly, if $\varphi^{(-\infty, 0]}(x) \subset V$, then $\alpha(x) \subset M_{i}$ for some $1 \leq i \leq n$. Now, for $j=0, \ldots, n$, we define

$$
\begin{aligned}
& I_{j}^{+}=I_{j}^{+}(V)=\left\{x \in V \mid \varphi^{[0, \infty)}(x) \subset V, \omega(x) \subset M_{j+1} \cup \cdots \cup M_{n}\right\}, \\
& I_{j}^{-}=I_{j}^{-}(V)=\left\{x \in V \mid \varphi^{(-\infty, 0]}(x) \subset V, \alpha(x) \subset M_{1} \cup \cdots \cup M_{j}\right\}, \\
& S_{j}^{*}=\left\{x \in S \mid \omega(x) \subset M_{j+1} \cup \cdots \cup M_{n}\right\}, \\
& S_{j}=\left\{x \in S \mid \alpha(x) \subset M_{1} \cup \cdots \cup M_{j}\right\} .
\end{aligned}
$$


Moreover, if $\left(A, A^{*}\right)$ is an attractor-repeller pair for $S$, we set

$$
\begin{aligned}
I_{A^{*}}^{+} & =\left\{x \in V \mid \varphi^{[0, \infty)}(x) \subset V, \omega(x) \subset A^{*}\right\}, \\
I_{A}^{-} & =\left\{x \in V \mid \varphi^{(-\infty, 0]}(x) \subset V, \alpha(x) \subset A\right\} .
\end{aligned}
$$

In $[2,11]$, it is proved that $I_{j}^{+}$and $I_{j}^{-}$are compact and $\left(S_{j}, S_{j}^{*}\right)$ is an attractor-repeller pair for $S$. This fact allows us to prove our results for an attractor-repeller pair and then extend them to every Morse decomposition.

In order to define the concept of index pair, we follow [9, 11]. Given a compact pair $(N, L)$ with $L \subset N \subset X$, we define the induced semiflow on $N / L$ by

$$
\varphi_{\#}^{t}: N / L \rightarrow N / L, \quad \varphi_{\#}^{t}(x)= \begin{cases}\varphi^{t}(x) & \text { if } \varphi^{[0, t]}(x) \subset N-L, \\ {[L]} & \text { otherwise. }\end{cases}
$$

In [9], it is proved that $\varphi_{\#}^{t}$ is continuous if and only if

(i) $L$ is positively invariant relative to $N$, that is,

$$
x \in L, t \geq 0, \quad \varphi^{[0, t]}(x) \subset N \Longrightarrow \varphi^{[0, t]}(x) \subset L,
$$

(ii) every orbit which exits $N$ goes through $L$ first, that is,

$$
x \in N, \quad \varphi^{[0, \infty)}(x) \not \subset N \Longrightarrow \exists t \geq 0, \quad \varphi^{[0, t]}(x) \subset N, \quad \varphi^{t}(x) \in L,
$$

or equivalently if $x \in N-L$, then there is a $t>0$ such that $\varphi^{[0, t]}(x) \subset N$.

DEFINITION 2.1. An index pair for an isolated invariant set $S \subset X$ is a compact pair $(N, L)$ in $X$ such that $\overline{N-L}$ is an isolating neighborhood for $S$ and the semiflow $\varphi_{\#}^{t}$ induced by $\varphi^{t}$ is continuous.

In $[1,2,9,11]$, it has been shown that every isolated invariant set $S$ admits an index pair $(N, L)$ and the homotopy type of the pointed space $N / L$ is independent of the choice of the index pair. The Conley index of $S$ is the homotopy type of $(N / L,[L])$.

Note 2.2. We will not distinguish between $N-L$ and $N / L-\{[L]\}$.

DEFINITION 2.3. An index pair $(N, L)$ is called regular if the exit time map defined by

$$
\boldsymbol{T}_{+}: N \rightarrow[0,+\infty], \quad \boldsymbol{T}_{+}(x)= \begin{cases}\sup \left\{t \mid \varphi^{[0, t]}(x) \subset N-L\right\} & \text { if } x \in N-L, \\ 0 & \text { if } x \in L,\end{cases}
$$

is continuous.

Proposition 2.4. An index pair $(N, L)$ is regular provided that $\varphi^{[0, t]}(x) \not \subset \overline{N-L}$ for every $x \in L$ and $t>0$. 
The above result provides a criterion for the regularity of index pairs. The reader is referred to [11] for the details about regular index pairs and the proof of this useful criterion.

DEFINITION 2.5. Let $S$ be an isolated invariant set with a Morse decomposition $\left\{M_{i}\right\}_{i=1}^{n}$. An index filtration is a sequence $N_{0} \subset N_{1} \subset \cdots \subset N_{n}$ of closed subsets of $X$ such that $\left(N_{k}, N_{k-1}\right)$ is an index pair for $M_{k}$ for every $1 \leq k \leq n$ and $\left(N_{n}, N_{0}\right)$ is an index pair for $S$. When each $\left(N_{k}, N_{k-1}\right)$ is regular, then the above filtration is called a regular index filtration.

It is well known that every Morse decomposition admits an index filtration [11]. We desire to show that every Morse decomposition admits a regular index filtration.

3. Conley's fundamental theorem. In this section, we construct a Lyapunov function on an index pair by modifying Conley's original argument $[1,10]$. The key point is that one should work with $I_{A^{*}}^{+}$and $[L] \cup I_{A}^{-}$instead of the attractor-repeller pair $\left(A, A^{*}\right)$. The following lemma is the main idea in the proof of the continuity of Conley's Lyapunov function.

LEMMA 3.1. Let $S$ be an isolated invariant set with an attractor-repeller pair $\left(A, A^{*}\right)$, an index pair $(N, L)$, and the isolating neighborhood $V=\overline{N-L}$. If $B$ is a compact subset of $N / L-I_{A^{*}}^{+}$and $U$ is a neighborhood of $[L] \cup I_{A}^{-}$, then there exists $T \in \mathbb{R}^{+}$such that $\varphi_{\#}^{[T,+\infty)}(B) \subset U$.

Proof. We may assume that $U$ is a compact neighborhood of $[L] \cup I_{A}^{-}$with $U \cap$ $I_{A^{*}}^{+}=\emptyset$. Now, suppose that there are $x_{n} \in B$ and $t_{n} \rightarrow \infty$ such that $\varphi_{\#}^{t_{n}}\left(x_{n}\right) \notin U$. Since $B$ is compact, we may choose $x_{n}$ 's in $N-L$ so that $x_{n} \rightarrow x \in B$. It is easy to see that $\varphi^{[0,+\infty)}(x) \subset N-L$. Since $B \cap I_{A^{*}}^{+}=\emptyset$, we have $\omega(x) \subset A$, hence there is a $t \in \mathbb{R}^{+}$such that $\varphi^{[t,+\infty)}(x) \in \stackrel{\circ}{U}$. Since $x_{n} \rightarrow x$, there are $t_{n}^{\prime} \in\left[t, t_{n}\right]$ such that $t_{n}^{\prime}-t \rightarrow \infty, \varphi^{\left[t, t_{n}^{\prime}\right)}\left(x_{n}\right) \subset \stackrel{\circ}{U}$, and $\varphi^{t_{n}^{\prime}}\left(x_{n}\right) \in \partial U$ for every sufficiently large $n \in \mathbb{N}$. Therefore, the sequence $\varphi^{t_{n}^{\prime}}\left(x_{n}\right)$ has a limit point $y \in \partial U$ such that $\varphi^{(-\infty, 0]}(y) \subset U \cap(N-L)$ and $y \in \omega(B) \subset S$. Thus, $\alpha(y) \subset A$, which means that $y \in I^{-}(A)$. This contradicts $y \in \partial U$.

THEOREM 3.2. Let $S$ be an isolated invariant set with an attractor-repeller pair $\left(A, A^{*}\right)$ and an index pair $(N, L)$. There exists a continuous function $g: N / L \rightarrow[0,1]$ such that

(i) $g^{-1}(0)=[L] \cup I_{A}^{-}$and $g^{-1}(1)=I_{A^{*}}^{+}$,

(ii) $g\left(\varphi_{\#}^{t}(x)\right)<g(x)$ for every $x \notin[L] \cup I_{A}^{-} \cup I_{A^{*}}^{+}$and $t \in \mathbb{R}^{+}$.

Proof. Let $\rho: N / L \rightarrow[0,1]$ be a continuous function with $\rho^{-1}(0)=[L] \cup I_{A}^{-}$and $\rho^{-1}(1)=I_{A^{*}}^{+}$. We define $h: N / L \rightarrow[0,1]$ by $h(x)=\sup _{t \geq 0} \rho\left(\varphi_{\#}^{t}(x)\right)$. It is not hard to see that $h^{-1}(0)=[L] \cup I_{A}^{-}, h^{-1}(1)=I_{A^{*}}^{+}$. We show that $h$ is upper-semicontinuous. For every $x \in N / L$ and $\epsilon>0$, there is a $t \in \mathbb{R}^{+}$such that $\rho\left(\varphi_{\#}^{t}(x)\right)>h(x)-\epsilon$. Now, there is a neighborhood $U$ such that $\rho\left(\varphi_{\#}^{t}(y)\right)>h(x)-\epsilon$ for every $y \in U$. Therefore, $h(y)>h(x)-\epsilon$ for every $y \in U$, which proves the upper-semicontinuity of $h$. As a result, $h$ is continuous in $h^{-1}(1)$. Now, suppose that $x \notin h^{-1}(1)=I_{A^{*}}^{+}$and $\epsilon<1-h(x)$. If we set $B=\rho^{-1}[0, h(x)+\epsilon]$ and $U=\rho^{-1}[0, h(x)+\epsilon)$ in the above lemma, we obtain 
a $T \in \mathbb{R}^{+}$with $\rho\left(\varphi_{\#}^{t}(y)\right)<h(x)+\epsilon$ for every $y \in B$ and $t \geq T$. Now, the by continuity of $\varphi_{\#}$, there exists an open set $V \subset N / L$ such that $x \in V$ and $\rho\left(\varphi_{\#}^{t}(y)\right)<h(x)+\epsilon$ for every $t \in[0, T]$ and $y \in V$. Therefore, $h(y)<h(x)-\epsilon$ for every $y \in U \cap V$, which shows that $h$ is lower-semicontinuous in $x$. Now, it is left to check that $g:=\int_{0}^{+\infty} e^{-t} h\left(\varphi_{\#}^{t}(x)\right) d t$ is the desired function.

THEOREM 3.3. Let $S$ be an isolated invariant set with an index pair $(N, L)$ and a Morse decomposition $\left\{M_{i}\right\}_{i=1}^{n}$. There is a continuous function $g: N / L \rightarrow[0, n+1]$ such that

(i) $g^{-1}(0)=[L]$ and $g\left(M_{i}\right)=i$ for every $1 \leq i \leq n$,

(ii) if $x \in N-L-\bigcup_{i=1}^{n} M_{i}$ and $t>0$, then $g\left(\varphi_{\#}^{t}(x)\right)<g(x)$.

Proof. Consider the attractor-repeller pairs $\left(S_{j}, S_{j}^{*}\right)$ for $0 \leq j \leq n$. By Theorem 3.2, there are continuous functions $g_{i}: N / L \rightarrow[0,1]$ with $g_{i}^{-1}(0)=[L] \cup I_{j}^{-}, g_{i}^{-1}(1)=I_{j}^{+}$, and $g_{j}\left(\varphi^{t}(x)\right)<g_{j}(x)$ for every $x \notin[L] \cup I_{j}^{-} \cup I_{j}^{+}$. Now, $g:=g_{0}+\cdots+g_{n}$ is the desired function.

COROLlARY 3.4. Every Morse decomposition admits a regular index filtration.

Proof. Let $f$ be the above Lyapunov function. If we set $N_{k}:=\pi^{-1}\left(f^{-1}[0, k+1 / 2]\right)$ for $0 \leq k \leq n$ and $N_{n}:=N$, then, by Proposition $2.4,\left(N_{k}, N_{k-1}\right)$ is a regular index pair for $M_{k}$, for every $1 \leq k \leq n$.

DEFINITION 3.5. Let $\varphi^{t}$ be a continuous flow on a compact metric space $X$. An $\epsilon$ chain for $\varphi^{t}$ is a sequence $x_{0}, \ldots, x_{n}$ in $X$ and $t_{1}, \ldots, t_{n}$ in $[1,+\infty)$ such that $d\left(\varphi^{t_{i}}\left(x_{i-1}\right)\right.$, $\left.x_{i}\right)<\epsilon$. A point $x \in X$ is called chain-recurrent if for every $\epsilon>0$, there is an $\epsilon$-chain with $x_{0}=x_{n}=x$. The set of all chain recurrent points for $\varphi^{t}$ is denoted by $R\left(\varphi^{t}\right)$.

It is not hard to check that $R\left(\varphi^{t}\right)$ is a closed invariant subset of $X$ containing the nonwandering set $\Omega\left(\varphi^{t}\right)$. In $[1,10]$, it has been shown that $R\left(\left.\varphi^{t}\right|_{R\left(\varphi^{t}\right)}\right)=R\left(\varphi^{t}\right)$ and $R\left(\varphi^{t}\right)=\bigcap\left(A \cup A^{*}\right)$, where the intersection is taken over all attractor-repeller pairs $\left(A, A^{*}\right)$ in $X$. It is also known that the number of all attractor-repeller pairs in a compact metric space is at most countable.

THEOREM 3.6. Let $S$ be an isolated invariant set with an index pair $(N, L)$. Then there is a continuous function $g: N / L \rightarrow[0,1]$ such that

(i) $g^{-1}(0)=[L]$ and $g\left(\varphi_{\#}^{t}(x)\right) \leq g(x)$ for every $x \in N / L$ and $t \geq 0$,

(ii) if $x \in N-L-R\left(\left.\varphi^{t}\right|_{S}\right)$ and $t \geq 0$, then $g\left(\varphi_{\#}^{t}(x)\right)<g(x)$.

Proof. Let $\left\{\left(A_{i}, A_{i}^{*}\right)\right\}_{i=1}^{\infty}$ be the sequence of all attractor-repeller pairs in $S$ including $(\emptyset, S)$ and $(S, \emptyset)$. Now, by Theorem 3.2, there are continuous functions $g_{i}: N / L \rightarrow[0,1]$ such that $g_{i}^{-1}(0)=[L] \cup I_{A_{i}}^{-}, g_{i}^{-1}=I_{A_{i}^{*}}^{+}$, and $g_{i}\left(\varphi_{\#}^{t}(x)\right)<g_{i}(x)$ for every $t \in \mathbb{R}^{+}$and $x \notin[L] \cup I_{A_{i}^{*}}^{+} \cup I_{A_{i}}^{-}$. Now, $g=\sum_{i=1}^{\infty} 2^{-i} g_{i}$ is the desired function.

The above result can be considered as a generalization of Conley's fundamental theorem of dynamical systems. A similar result for discrete dynamical systems can be obtained by following $[4,12]$.

ACKNOWLEDGMENT. The author would like to thank the Institute for Studies in Theoretical Physics and Mathematics (IPM) for supporting this research. 


\section{REFERENCES}

[1] C. Conley, Isolated Invariant Sets and the Morse Index, CBMS Regional Conference Series in Mathematics, vol. 38, American Mathematical Society, Rhode Island, 1978.

[2] C. Conley and E. Zehnder, Morse-type index theory for flows and periodic solutions for Hamiltonian equations, Comm. Pure Appl. Math. 37 (1984), no. 2, 207-253.

[3] J. Franks, A variation on the Poincaré-Birkhoff theorem, Hamiltonian Dynamical Systems (Boulder, Colo, 1987), Contemp. Math., vol. 81, American Mathematical Society, Rhode Island, 1988, pp. 111-117.

[4] J. Franks and D. Richeson, Shift equivalence and the Conley index, Trans. Amer. Math. Soc. 352 (2000), no. 7, 3305-3322.

[5] M. Hurley, Chain recurrence and attraction in noncompact spaces, Ergodic Theory Dynam. Systems 11 (1991), no. 4, 709-729.

[6] _ Noncompact chain recurrence and attraction, Proc. Amer. Math. Soc. 115 (1992), no. $4,1139-1148$.

[7] _ Chain recurrence, semiflows, and gradients, J. Dynam. Differential Equations 7 (1995), no. 3, 437-456.

[8] L_ Lyapunov functions and attractors in arbitrary metric spaces, Proc. Amer. Math. Soc. 126 (1998), no. 1, 245-256.

[9] J. W. Robbin and D. Salamon, Dynamical systems, shape theory and the Conley index, Ergodic Theory Dynam. Systems 8* (1988), no. Charles Conley Memorial Issue, 375393.

[10] C. Robinson, Dynamical Systems, Studies in Advanced Mathematics, CRC Press, Florida, 1995.

[11] D. Salamon, Connected simple systems and the Conley index of isolated invariant sets, Trans. Amer. Math. Soc. 291 (1985), no. 1, 1-41.

[12] A. Szymczak, The Conley index for discrete semidynamical systems, Topology Appl. 66 (1995), no. 3, 215-240.

M. R. Razvan: Institute for Studies in Theoretical Physics and Mathematics, P.O. Box 193955746, Tehran, Iran

E-mail address: razvan@i pm. i r 


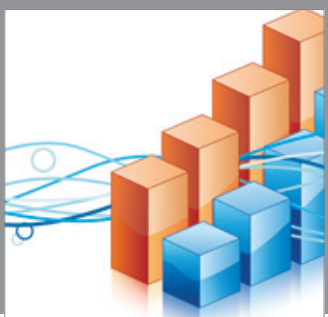

Advances in

Operations Research

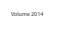

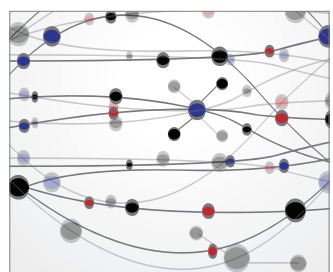

\section{The Scientific} World Journal
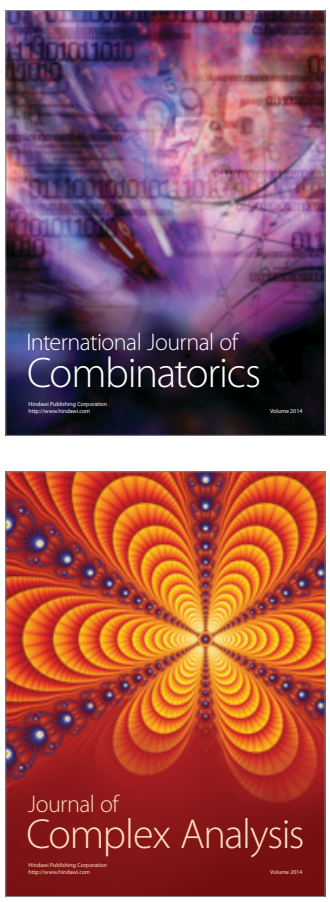

International Journal of

Mathematics and

Mathematical

Sciences
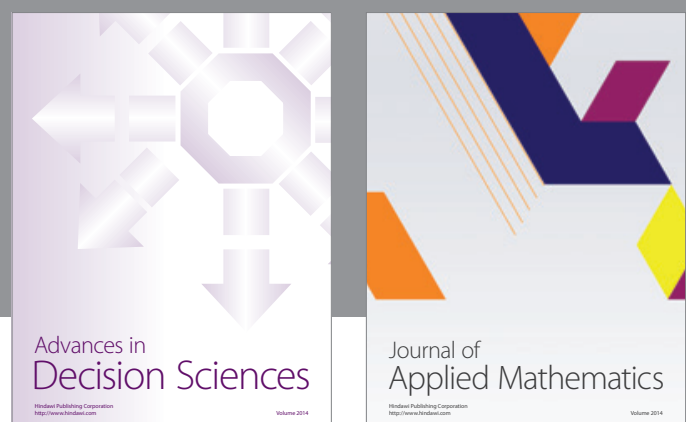

Journal of

Applied Mathematics
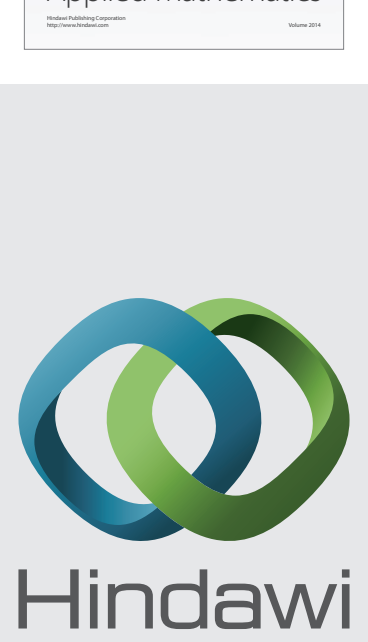

Submit your manuscripts at http://www.hindawi.com
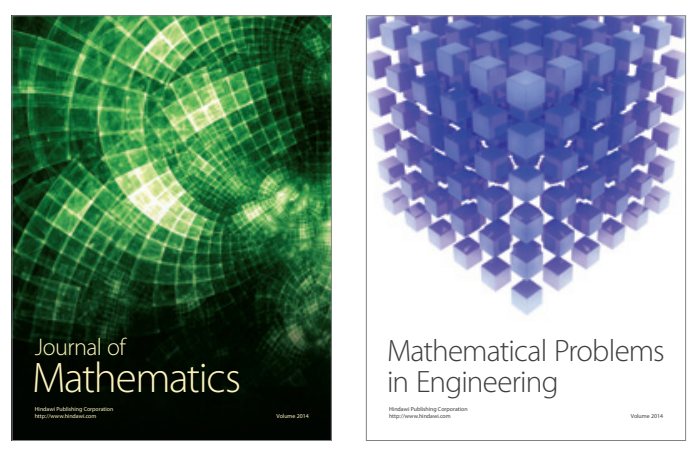

Mathematical Problems in Engineering
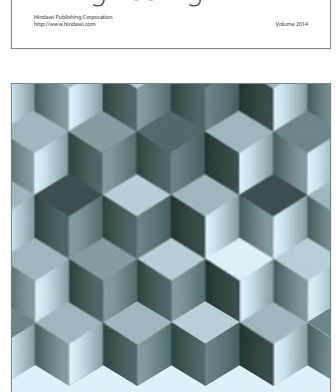

Journal of

Function Spaces
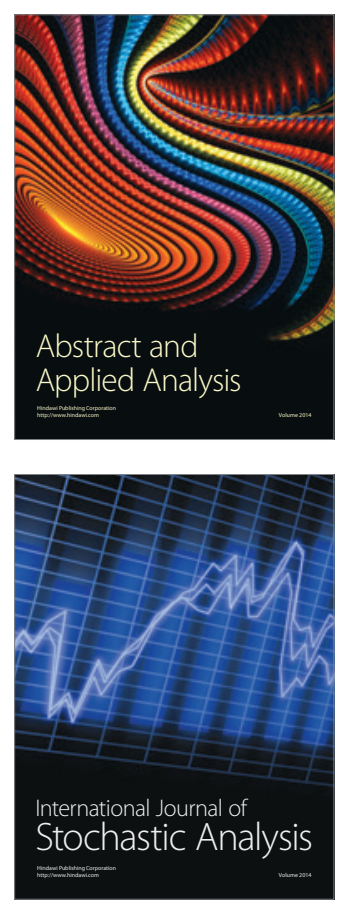

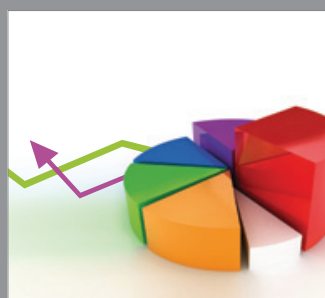

ournal of

Probability and Statistics

Promensencen
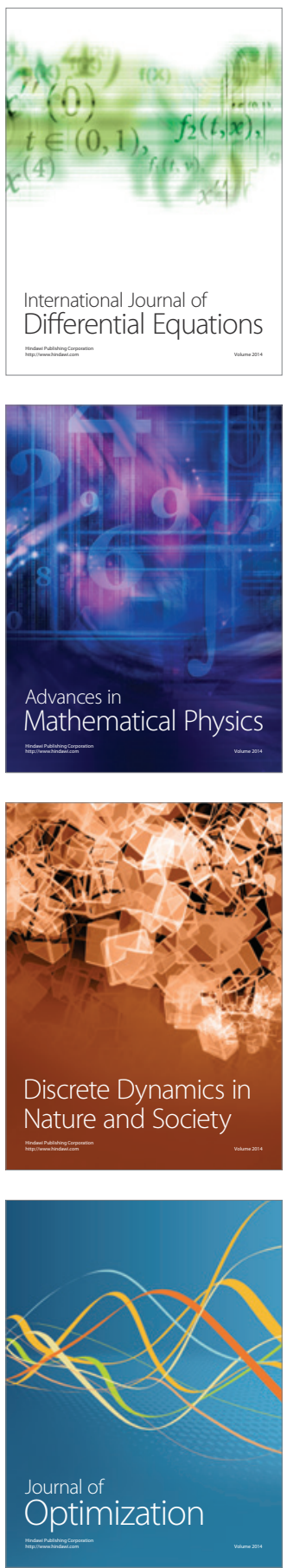